\title{
Structural flexibility in the solid state
}

\section{Len Barbour}

Department of Chemistry and Polymer Science, Stellenbosch University, South Africa

e-mail: ljb@sun.ac.za

In order to understand solid-gas inclusion processes at the molecular level it is important to correlate physico-chemical data (e.g. sorption isotherms and calorimetric analysis) with structural data. It is therefore desirable to carry out structural elucidation and calorimetric analysis under conditions that closely mimic those of the sorption/desorption experiments. However, the crystallographic analysis of samples under controlled gas environments poses significant technical challenges, particularly given the limited space associated with the sample compartment of standard commercial diffractometer. In this regard, an environmental gas cell has been developed in parallel with a pressure-programmed differential scanning calorimeter. Use of these complementary techniques has provided new insight into features such as pressure-induced phase transformations that give rise to inflections and hysteresis in sorption isotherms. The influence of guest molecules on aspects such as structural flexibility and changes in network interpenetration will be discussed. 